\title{
A critical evaluation of software for experiment development in research and teaching
}

\author{
DARRELL L. BUTLER \\ Ball State University, Muncie, Indiana
}

\begin{abstract}
This paper compares several software packages that allow users to create new computer-run experiments, but do not require that users be able to program. Three dimensions are considered: package requirements, ease of learning, and power and flexibility.
\end{abstract}

Almost 10 years ago, members of the Society for Computers in Psychology began having serious discussions about a major problem in undergraduate education: To implement new computer-controlled psychology experiments, undergraduates needed to have reasonably good computer programming skills, and most did not have these skills. One solution to this problem is to provide students with an intelligent experiment generator, that is, some software that students who do not know how to program can use with little or no training to create computercontrolled experiments. Several systems of this type have become available. Eamon (1982) described CEDATS, a set of BASIC programs for the Apple II that permitted students to set up, conduct, and analyze experiments. The same year, Poltrock and Foltz (1982) described APT, a set of Pascal programs for the Apple II that permitted students to set up and conduct experiments. More recently, Poltrock and Foltz (1988) have adapted their system to the IBM PC. Costin (1988) described a new experiment generator, MacLab, for the Macintosh computer, and Schneider (1988) described a new experiment generator, MEL, for the IBM PC.

Researchers and instructors are now in the enviable position of being able to choose from among these packages. The purpose of this paper is to begin to compare them and analyze their uses. For this review, I worked with CEDATS on Apple II+ and Apple IIe, APT on a Zenith 159 (IBM PC compatible), MEL on an IBM PC, and MacLab on a Macintosh Plus. Readers should note APT runs similarly on the Apple II and the Zenith.

A number of factors must be considered in a fair comparison of these packages. First, their hardware requirements differ substantially. For those who have already made substantial hardware commitments, this may determine the package of choice. Second, there is great variability in the ease with which the packages can be learned. This is an especially important factor if these packages

\footnotetext{
The author would like to thank the authors of the software for sup plying copies of the software and manuals and for their help. Also, thanks to Ann Fischer and Steve Jones, two undergraduates who helped collect the information presented in this paper. Requests for reprints should be sent to the author at the Department of Psychological Sciences, Ball State University, Muncie, IN 47306.
}

are being considered for use in undergraduate courses. Third, the packages differ greatly in the types of experiments that can be run. For some experiments, only one of these packages may be useful.

\section{PACKAGE DESCRIPTIONS}

\section{CEDATS}

CEDATS requires a $48 \mathrm{~K}$ Apple II $(64 \mathrm{~K}$ is recommended) and one disk drive, and is contained on one double-sided floppy diskette. The package is written primarily in Applesoft, although some routines are written in machine code. A variety of sample experiments are included. Two manuals are provided: A 42-page student guide and an 118-page instructor's guide. Although the system is relatively easy to use, the manuals are needed. Cost is $\$ 70$. CEDATS is presently being revised and enhanced. The improved version may be available by the time this article is published.

\section{APT}

There are two versions of APT. One requires a 64K Apple II with either a Mountain Hardware Clock or a Timemaster II H.O. clock. The Pascal operating system (not included with the package) is also required. The other version requires a $256 \mathrm{~K}$ IBM PC or clone and the MSDOS operating system. The package, including sample experiments, is provided on one diskette. One 25-page manual is provided. Cost is $\$ 100$ per package; source code is available for an additional $\$ 100$. There were no specific plans for developing new versions at the time of this writing.

\section{MEL}

It is difficult to develop software using MEL unless one has a 640K IBM PC or clone with a hard disk drive and a floppy disk drive. To run subjects, MEL requires a $384 \mathrm{~K}$ (640K is recommended) IBM PC or clone with a floppy disk drive. Graphics cards and color monitors are supported and are desirable for some kinds of data analysis and experiments. The evaluation version (a prerelease version) of the package came on five diskettes. Some sample experiments were included. Three documents were provided: a 15-page introduction, a 15-page user's guide, 
and a 157-page language reference manual. The manuals were not in final form; it is expected that the combined length of the final manuals will be over 400 pages. The manuals are needed. Cost depends upon several factors; the maximum price for research use is $\$ 495$ and the maximum price for instructional use is $\$ 295$.

\section{MacLab}

MacLab requires a 512K Macintosh. A hard disk drive is recommended, but not required. The programs come on two diskettes; one is a demonstration disk. Two manuals are included: a 25-page manual on MacLab and a 13-page manual on ScriptToPaint, a useful utility package accompanying MacLab. A user's manual designed for first-time Macintosh users is in preparation and should be available by the time this article is published. Cost is $\$ 200$.

\section{EASE OF LEARNING}

One of my students and I used each of these packages and found APT the easiest to learn, CEDATS second, MacLab third, and MEL the most difficult.

I first spent $3 \mathrm{~h}$ with each package. APT proved easy to learn. In fact, after a short period of time, I was able to write short experiments and discover limitations of the program. It took about $1 \mathrm{~h}$ to learn to create and run simple experiments with CEDATS, and I needed the manual to clarify some concepts. MacLab was a little harder to learn. It took me about $2.5 \mathrm{~h}$ to create my first onestimulus experiment, and the manuals were an absolute necessity. After installing MEL on a hard disk, I spent $3 \mathrm{~h}$ using the on-line tutorials; at the end of this time, I was beginning to understand how to use the system. The tutorials tell the user what to do and then permit only a preset sequence of keypresses; all other keypresses are locked out. Near the end of the tutorials, I was fairly certain I recognized a mistake in one of the field specifications; unfortunately, the tutorial would not let me change it. I ran the program as created and it bombed. (Note that this "bug" occurred in a prerelease version of the package, and such bugs will be removed before the package is sold. If any new bugs are discovered in the documentation later, the author will correct it and make a new version available as rapidly as possible.) It took a few more hours of working with MEL before I could successfully create simple experiments, and several more hours of working and reading the manuals before MEL became clear.

A senior honors student who is familiar with some applications programs on Apple II and Macintosh computers attempted to create simple experiments on each of the packages. She initially spent about an hour with each package. Then she and I talked about the package and she returned to it, spending $3 \mathrm{~h}$ on each package. The only package she was able to use after $1 \mathrm{~h}$ of exposure was APT, which she found very easy to use, even though she had no previous experience on an IBM PC. In $30 \mathrm{~min}$, she had created a short questionnaire experiment and was ready to run a subject. She needed the manual only to understand the method used for randomizing stimuli. The next easiest package for her was CEDATS, which presented a few problems. First, she did not have the cap lock key pressed on the Apple IIe and the program did not identify some of her answers to questions. She also had two problems with terminology and had to refer to the manual. She was not able to create anything with either MEL or MacLab within the 3-h limit.

All of the packages follow reasonably good program design (see Butler, 1986b), and therefore should be relatively easy to learn. The differences in ease of learning appear to be primarily attributable to differences in the power of the programs. The more powerful programs are harder to learn because they have many more options and concepts to master.

\section{POWER AND FLEXIBILITY}

MEL was the most powerful and flexible, followed by MacLab, APT, and CEDATS. For a complete description of each package, see the up-to-date manuals on the packages and/or articles describing their capabilities (e.g., Costin, 1988; Eamon, 1982; Poltrock \& Foltz, 1988; Schneider, 1988). A few major characteristics are described below.

One of the most important differences among the programs is in the type of stimuli that can be presented. All of the packages allow stimuli to be text screen characters, that is, the characters that can be generated by pressing keys on the keyboard. MEL and MacLab can also present graphics. In addition, MEL can present tones in the 1 to $20000 \mathrm{~Hz}$ range for music and other kinds of auditory research. The packages would require programming to permit presentation of other kinds of stimuli. Theoretically, any of the packages could be adapted to drive slide projectors and other devices. MEL has the greatest flexibility in this regard.

A second difference is in whether stimuli must be defined as single units or whether parts of stimuli can be defined and the package will combine them for the researcher. All of the packages permit a sequence of screens to be defined as a stimulus condition. In this regard, CEDATS is the least flexible because researchers are basically limited to two screens. MEL is unique in that not only does it permit sequences of screen displays to define stimuli, but parts of each screen and durations of displays can be treated as stimulus variables. Thus words in sentences and parts of pictures can be defined and substituted systematically for one another. Because of this feature, stimuli for many cognitive experiments can be defined faster with MEL than with any of the other packages.

Another major difference among the programs is in the types of responses allowed. All of the packages allow single-key input from a keyboard and store keypresses (or accuracy) as well as response time. In addition, APT, 
MEL, and MacLab permit string input. MEL provides software support for other types of responses (e.g., voice key and mouse), but some programming is necessary to use this option. MacLab permits the mouse key to be used for input.

The programs differ substantially with respect to ease of data analysis. All of the programs provide easy access to individual subject data files. CEDATS will compute means of conditions and a few other descriptive statistics. Furthermore, a program, REFORMAT, is provided that creates files usable by statistics programs such as HSD ANOVA II (not provided with CEDATS). APT provides two data analysis programs. Basically, they provide the same summaries as does CEDATS. For other analyses, users have to write their own analysis programs, or some simple programs to change data into a form usable by their statistics package. MacLab writes data into a text file, but provides no analysis programs. MEL contains the most sophisticated analysis routines. Descriptive statistics can be obtained by subject, trials, block, and so forth. Data can be "filtered," that is, unreasonable data points or subjects can be excluded. ANOVAs and correlations can be computed. In addition, graphs and other summaries can be obtained. MEL also provides utilities to write the data into structures usable by a variety of statistics programs.

One statistic that is indicative of the power of these packages is the number of published studies that could have been run using each of them. To evaluate the experiment-generator packages I used the first 11 issues of Perception \& Psychophysics, 1987. A total of 225 experiments were reported in these issues; of these, 148 used computers for stimulus presentation, response collection, or both. MEL could have been used for 104 experiments (70\% of those using computers), MacLab could have been used for 77 experiments (52\% of those using computers), and either CEDATS or APT could have been used for 31 experiments ( $21 \%$ of those using computers). Although these statistics provide a useful comparison, they should not be interpreted too strictly. For one thing, Perception \& Psychophysics is not representative of all journals. In an earlier study (Butler, 1986a), I found that across journals, $37 \%$ of human experiments were automated. Certain journals, such as the Journal of Verbal Learning \& Verbal Behavior, had a very small percentage of automated experiments, about $15 \%$; other journals, such as Psychological Review, had a relatively high percentage, about $62 \%$. In the first 11 issues of the 1987 Perception \& Psychophysics, $66 \%$ of the experiments were automated. Also, Perception \& Psychophysics carries a relatively large percentage of experiments using stimuli other than text. Such stimuli cannot be presented by CEDATS or APT. In addition, my judgments about necessity and capability may affect the accuracy of the percentages reported above for the experiment-generating packages. For some experiments reported in the journal, a tachistoscope was used when it may not have been necessary. The computer could have replaced all pencil and paper tests, as well as tape recorders and other kinds of equipment. Perhaps the major problem with estimating whether an experiment could have been run using one of these packages involved deciding whether a piece of sophisticated hardware (e.g., a scleral reflectance eye monitor) could be easily handled by one or more of these packages. With modest amounts of programming to permit the packages to handle special interfaces, the capabilities of these packages would be boosted significantly above the percentages provided above.

\section{CONCLUSION}

CEDATS and APT are easy-to-use experiment generators, and they are similar in many respects. Perhaps the greatest differences are that APT is a little easier to use the first time and has a little more flexibility in regard to generating sequential stimuli, whereas CEDATS has better data analysis provisions and is less expensive. MacLab and MEL are not as easy to learn as are CEDATS and APT; if they are to be used at the undergraduate level, an experienced user should be available to help students. Once a large base of experiments was available, MEL (and probably MacLab) would be easy to alter to run new experiments. After all, most undergraduate students usually run only slightly altered versions of standard paradigms, and such changes would be easy for a lab assistant to implement.

I think all of these packages are outstanding. I am especially impressed with MEL. My initial evaluation of MEL is that it is powerful enough and flexible enough to have run all of the cognitive experiments I have done over the past 7 years, with the exception of two sorting studies. If I had had MEL when I began as an assistant professor, I believe it would have saved me hundreds of hours of programming.

All of these software packages are useful research tools, suitable for use in professional laboratories, and they are the kinds of tools that students should be encouraged to use.

\section{REFERENCES}

BUTLER, D. L. (1986a). Automation of instructions in human experiments. Perceptual \& Motor Skills, 63, 435-440.

BUTLER, D. L. (1986b). Integrating statistical software into laboratories and laboratory courses. Behavior Research Methods, Instruments, \& Computers, 18, 241-244.

Costin, D. (1988). MacLab: A Macintosh system for psychology labs. Behavior Research Methods, Instruments, \& Computers, 20, 197-200.

EAMON, D. B. (1982). CEDATS: A cognitive experimental design and testing system. Behavior Research Methods \& Instrumentation, 14, 142-145.

Poltrock, S. E., \& FolTz, G. S. (1982). An experimental psychology laboratory system for the Apple II microcomputer. Behavior Research Methods \& Instrumentation, 14, 103-108.

Poltrock, S. E., Foltz, G. S. (1988). APT PC and APT II: Experiment development systems for the IBM PC and Apple II. Behavior Research Methods, Instruments, \& Computers, 20, 201-205.

Schneider, W. (1988). Micro Experimental Laboratory: An integrated system for IBM PC compatibles. Behavior Research Methods, Instruments, \& Computers, 20, 206-217. 